\title{
Starch Synthesis in Ostreococcus tauri: The Starch-Binding Domains of Starch Synthase III-B Are Essential for Catalytic Activity
}

\author{
Julieta Barchiesi ${ }^{1 \dagger}$, Maria Belen Velazquez ${ }^{1 \dagger}$, Nicolas Palopoli ${ }^{2}$, Alberto A. Iglesias ${ }^{3}$, \\ Diego F. Gomez-Casati ${ }^{1}$, Miguel Angel Ballicora ${ }^{4}$ and Maria Victoria Busi ${ }^{1 *}$
}

${ }^{1}$ Centro de Estudios Fotosintéticos y Bioquímicos (CEFOBI-CONICET), Universidad Nacional de Rosario, Rosario, Argentina, ${ }^{2}$ Departamento de Ciencia y Tecnología, Universidad Nacional de Quilmes and CONICET, Bernal, Argentina, ${ }^{3}$ Laboratorio de Enzimología Molecular, Instituto de Agrobiotecnología del Litoral (CONICET- Universidad Nacional del Litoral) and Facultad de Bioquímica y Ciencias Biológicas, Santa Fe, Argentina, ${ }^{4}$ Department of Chemistry and Biochemistry, Loyola University Chicago, Chicago, IL, United States

OPEN ACCESS

Edited by:

Yasunori Nakamura,

Akita Prefectural University, Japan

Reviewed by:

Jose Antonio Cuesta-Seijo,

Carlsberg Research Laboratory,

Denmark

Naoko Fujita,

Akita Prefectural University, Japan

*Correspondence:

Maria Victoria Busi

busi@cefobi-conicet.gov.ar

tThese authors have contributed equally to this work

Specialty section: This article was submitted to

Plant Metabolism

and Chemodiversity,

a section of the journal

Frontiers in Plant Science

Received: 18 July 2018 Accepted: 02 October 2018 Published: 25 October 2018

Citation:

Barchiesi J, Velazquez MB, Palopoli N, Iglesias AA, Gomez-Casati DF, Ballicora MA and Busi MV (2018)

Starch Synthesis in Ostreococcus

tauri: The Starch-Binding Domains of Starch Synthase III-B Are Essential

for Catalytic Activity.

Front. Plant Sci. 9:1541.

doi: 10.3389/fpls.2018.01541
Starch is the major energy storage carbohydrate in photosynthetic eukaryotes. Several enzymes are involved in building highly organized semi-crystalline starch granules, including starch-synthase III (SSIII), which is widely conserved in photosynthetic organisms. This enzyme catalyzes the extension of the $\alpha-1,4$ glucan chain and plays a regulatory role in the synthesis of starch. Interestingly, unlike most plants, the unicellular green alga Ostreococcus tauri has three SSIII isoforms. In the present study, we describe the structure and function of OsttaSSIII-B, which has a similar modular organization to SSIII in higher plants, comprising three putative starch-binding domains (SBDs) at the N-terminal region and a C-terminal catalytic domain (CD). Purified recombinant OsttaSSIII-B displayed a high affinity toward branched polysaccharides such as glycogen and amylopectin, and to ADP-glucose. Lower catalytic activity was detected for the CD lacking the associated SBDs, suggesting that they are necessary for enzyme function. Moreover, analysis of enzyme kinetic and polysaccharide-binding parameters of site-directed mutants with modified conserved aromatic amino acid residues W122, Y124, F138, Y147, W279, and W304, belonging to the SBDs, revealed their importance for polysaccharide binding and SS activity. Our results suggest that OT_ostta13g01200 encodes a functional SSIII comprising three SBD domains that are critical for enzyme function.

Keywords: starch, starch synthase, starch-binding domains, microalgae, enzyme regulation

\section{INTRODUCTION}

Starch plays a key role in the life cycle of plants as the primary form of carbohydrate storage for chemical energy. Additionally, it has acquired immense value as a vital source of energy and as a precursor of new biodegradable materials (Ball and Morell, 2003; Sticklen, 2006). Starch accumulates as semicrystalline granules with a complex structure and organization composed of two polymers, amylose (AM) and amylopectin (AP). In both polymers, glucose is connected by $\alpha-1,4$ glycosidic bonds, forming linear chains, and $\alpha-1,6$ bonds in the so-called branch points. 
$\mathrm{AM}$ is a mostly linear polymer, accounting for between 15 and $35 \%$ of the weight of granules. AP has a larger number of branches (5\%) and is the dominant component, accounting for $65-85 \%$ of granules (Nakamura, 2002; Zeeman et al., 2010; Pfister and Zeeman, 2016).

There are four biochemical steps in starch synthesis (substrate activation, chain elongation, chain branching, and chain debranching) carried out by different enzymes (Preiss et al., 1991; Zeeman et al., 2010). In green plants, soluble and granule-bound starch synthases (SS and GBSS, respectively), which mainly utilize ADP-glucose (ADPGlc), are derived from the bacterial symbiont that gave rise to each branch of the Archeplastida. By contrast, SSs from red algae and glaucophytes mainly utilize UDP-glucose (UDPGlc) and are soluble forms derived from the host, while GBSS-like proteins are derived from the symbiont (Patron and Keeling, 2005; Deschamps et al., 2008a,b,c).

The first isolation of Ostreococcus tauri dates from 1994 in a lagoon in Thau, France. This picoalga is a member of the Prasinophyceae, which places it in a key phylogenetic position as a study model because it shares the common ancestor of Chorophyta that currently dominate terrestrial photosynthesis. O. tauri lacks a cell wall and flagella, and contains a large nucleus, a single chloroplast, a mitochondrion, and a Golgi apparatus in a reduced cytoplasmic compartment (Demir-Hilton et al., 2011). Although $O$. tauri constitutes the smallest eukaryotic cell and possesses the smallest genome of a eukaryotic photosynthetic organism described to date, it contains more SSIII-like genes (three copies) than any other algae or plant species (e.g., Arabidopsis thaliana has only one copy) (Deschamps et al., 2008c). Thus, the genome of O. tauri encodes three SSIIIs (SSIII-A, SSIII-B, and SSIII-C) of which only SSIII-C has been characterized (Barchiesi et al., 2017). Interestingly, the O. tauri genome lacks genes related to yeast or mammalian glycogenin. Thus, the conservation throughout evolution of the three SSIII isoforms, and the absence of SSIV, could be related to the presence of a single starch granule in this alga with a particular partitioning and propagation mechanism (Ral et al., 2004; Deschamps et al., 2008c).

It is important to note that SSs in both plants and algae have a modular organization. The C-terminal region of all SS isoforms includes a conserved glycosyltransferase (GT) domain, while the $\mathrm{N}$-terminal region includes starch-binding domains (SBD), a specific group of carbohydrate-binding modules (CBM). The generic term CBM refers to a contiguous amino acid sequence with a distinctive fold within an enzyme that may or may not be catalytically active with carbohydrate substrates (Penninga et al., 1996; Boraston et al., 2004; Guillén et al., 2010). The ability of CBMs to bind polysaccharides can be attributed, at least partially, to several aromatic residues that are assembled on a hydrophobic surface. Specifically, SBDs have gained the evolutionary advantage of enabling CBMs to disrupt the structure of the substrate more efficiently due to the presence of two polysaccharide-binding sites (Tormo et al., 1996; Southall et al., 1999). The SSIII isoform of A. thaliana (ArathSSIII), containing a GT5 domain ${ }^{1}$ at its

${ }^{1}$ http://www.cazy.org/
C-terminal end and three in tandem SBDs belonging to the CBM53 family in its N-terminal region (designated D1-D2-D3), demonstrated the importance and functionality of an SBD in a biosynthetic enzyme (Palopoli et al., 2006; Busi et al., 2008; Valdez et al., 2008, 2011). The CBM53 family currently includes 96 entries in the CAZy database (see footnote 1), and it is widely distributed in archaea, bacteria, and eukaryotes. Threedimensional structures of numerous CBMs are available for nine families, but structures of CBM45 and CBM53 families have not yet been reported.

Herein, we describe the cloning and expression of the OsttaSSIII-B gene, and purification and characterization of the recombinant protein produced in Escherichia coli. Our results show that the OsttaSSIII-B isoform is a modular protein with three N-terminal SBDs and a C-terminal CD, being the SBDs important for its catalytic activity. The purified recombinant protein displayed a high affinity toward branched polysaccharides such as glycogen and AP. Analysis of enzyme kinetic and polysaccharide-binding parameters of OsttaSSIII-B site-directed mutants with altered conserved aromatic amino acid residues W122, Y124, F138, Y147, W279, and W304, belonging to the SBDs, revealed their importance for polysaccharide binding and SS activity.

\section{MATERIALS AND METHODS}

\section{Strains and Culture Media}

Escherichia coli strains XL1 Blue (endA1, gyrA46, hsdR17, lac ${ }^{-}$, recA1, relA1, supE44, thi-1, $\mathrm{F}^{\prime}\left[\right.$ proAB $B^{+}$, lacI $I^{\mathrm{q}}$ lacZ $\Delta M 15$, $\operatorname{Tn} 10\left(\right.$ tet $\left.\left.^{\mathrm{r}}\right)\right]$ ) and BL21 (DE3) pLysS (F-ompT gal dcm hsd $S_{\mathrm{B}}\left[\mathrm{r}_{\mathrm{B}}{ }^{-}\right.$ $\left.\mathrm{m}_{\mathrm{B}}{ }^{-}\right] \lambda[\mathrm{DE} 3]$ pLysS $\left.\left[\mathrm{Cm}^{\mathrm{r}}\right]\right)$ were purchased from Stratagene (La Jolla, CA, United States). Bacterial cells were grown at $37^{\circ} \mathrm{C}$ in Luria Bertani (LB) medium containing the appropriate antibiotics.

\section{Protein Sequence Analysis}

Domains of OsttaSSIII-B were predicted using the KEGG Sequence Similarity Database (SSDB) Motif Search server (Kanehisa et al., 2017). Alignment of OsttaSSIII-B, OsttaSSIIIC, ArathSSIII-CD, AgrtuGS, and EsccoGS amino acids sequences was performed using the ClustalW algorithm of Unipro UGENE v.1.10.4 (Okonechnikov et al., 2012) with default parameters.

\section{Protein Structure Modeling}

Structural models of the D1, D2, and D3 domains were described previously (Barchiesi et al., 2015). These were built by comparative modeling using Modeller v9.13 (Sali, 1995) with templates CBM25-1 of beta/alpha-amylase from Paenibacillus polymyxa (PDB code: 2LAA), CBM25 from Bacillus halodurans amylase (PDB code: $2 \mathrm{C} 3 \mathrm{~V}$ ), and the glycogen-binding domain of the AMP-activated protein from Rattus norvegicus (PDB code: $1 \mathrm{Z0N}$ ), respectively (Kawazu et al., 1987; Polekhina et al., 2005; Boraston et al., 2006). 


\section{Cloning, Expression, and Purification of OsttaSSIII-B and OsttaSSIII-B CD}

The OsttaSSIII-B gene (OT_ostta13g01200) was cloned from O. tauri genomic DNA (kindly provide by Dr. Evelyne Derelle) into KpnII and HindIII restriction enzyme sites of the pRSETC vector (Invitrogen, CA, United States) using standard molecular biology procedures and primers OtSSIII-B Nt Fwd (TGCGGTACCCGCGTCGGTGGAG) and OtSSIII-B Ct Rev (CTAAGCTTTTACCGACCGATCATC). OsttaSSIII-B CD was amplified from pRSET-C::OsttaSSIII-B using primers OtSSIII-B CD Fwd (AAGGTACCTCTTCACGTCTGTCAC) and OtSSIII-B Ct Rev, and cloned into KpnII and HindIII sites of pRSET-C ( KpnI and HindIII sites are underlined). The expression vectors pRSETC::OsttaSSIII-B and pRSET-C::OsttaSSIII-B CD (both containing an $\mathrm{N}$-terminal His-tag sequence) were used to transform E. coli BL21 (DE3) pLysS, and cells were grown at $37^{\circ} \mathrm{C}$ for $3 \mathrm{~h}$, then induced with $0.5 \mathrm{mM}$ isopropyl $\beta$ - $D$-1-thiogalactopyranoside (IPTG) and incubated at $20^{\circ} \mathrm{C}$ for at least $18 \mathrm{~h}$. Cells were harvested by centrifugation at $5000 \times g$ for $15 \mathrm{~min}$ at $4^{\circ} \mathrm{C}$, and the pellet was resuspended in $20 \mathrm{mM}$ TRIS- $\mathrm{HCl}(\mathrm{pH} 7.5)$. Cells were disrupted by sonication and centrifuged at 12,000 $\times g$ for $15 \mathrm{~min}$ at $4^{\circ} \mathrm{C}$. The homogenate was loaded onto a HiTrap chelating HP column (GE Healthcare BioSciences, Uppsala, Sweden) equilibrated with binding buffer ( $20 \mathrm{mM}$ TRIS-HCl $\mathrm{pH}$ 7.5, $20 \mathrm{mM}$ imidazole). The column was washed with 10-15 volumes of binding buffer, and each protein was eluted using a linear gradient of binding buffer and elution buffer $(20 \mathrm{mM}$ TRIS-HCl pH 7.5, 20-500 mM imidazole) (Barchiesi et al., 2017). The presence of OsttaSSIII-B (103.2 kDa) or OsttaSSIII-B CD $(56.3 \mathrm{kDa})$ in the eluted fractions was monitored by sodium dodecyl sulfate-polyacrylamide gel electrophoresis (SDS-PAGE) and Western blot analyses. Fractions containing the protein of interest were stored at $-40^{\circ} \mathrm{C}$ after addition of $20 \%(\mathrm{v} / \mathrm{v})$ glycerol.

\section{Construction, Expression, and Purification of Site-Directed Mutants}

OsttaSSIII-B mutants W122AY124A, F138A, Y147A, W279A, and W304A were obtained using the QuickChange II site-directed mutagenesis kit (Stratagene, La Jolla, CA, United States). The pRSET-C::OsttaSSIII-B vector was used as template for PCR amplification. The following primers (and their complements) were used (base substitutions in italics and underlined): OtB W122AY124A, GGTGAAGGGGACGTCGGCGGTGGCCGCCGATGTGAACG TG; OtB F138A, CGCGCGTTCTCGATGGATGCCGTGTTCA AGGGAGA; OtB Y147A, GAGACGGCGAGGCGGCCGAGAA GGACGAAG; OtB W279A, AGAACAACGACTGGGCGGTCG CCGACGTCC, and OtB W304A, CGTCGA AGAGTCG GCCGCGGATAACAACGAACAG. Mutations were confirmed by DNA sequencing (Macrogen, Korea). The resultant plasmids, named pRSET-C::OsttaSSIII-B W122AY124A, pRSET-C::OsttaSSIII-B F138A, pRSET-C::OsttaSSIII-B Y147A, pRSET-C::OsttaSSIII-B W279A, and pRSET-C::OsttaSSIII-B W304A (all containing an $\mathrm{N}$-terminal His-tag sequence) were used to transform E. coli BL21 (DE3) pLysS cells. Mutated recombinant proteins were expressed, purified, and stored at $-40^{\circ} \mathrm{C}$ as described above.

\section{Determination of Denaturation Temperature as an Indicator of Protein Stability}

Differential scanning fluorimetry (DSF) was performed on an Applied Biosystems real-time PCR instrument (Giuliani et al., 2008). The technique is based on the properties of some dyes that interact with proteins during thermal unfolding, resulting in an increase in fluorescence upon contact with the hydrophobic core of the protein. Thus, $4 \mu \mathrm{g}$ of wild-type (WT) or mutated protein was 5000-fold diluted with SYPRO Orange dye to a total volume of $25 \mu \mathrm{l}$ in $0.2 \mathrm{ml}$ optical 8-Cap Tube Strips (Life Technologies). Samples were heated from $15^{\circ} \mathrm{C}$ to $95^{\circ} \mathrm{C}$ with increments of $1^{\circ} \mathrm{C} / \mathrm{min}(\sim 1.5 \mathrm{~h})$ and fluorescence data were collected using a $550 \mathrm{~nm}$ filter in melting temperature (Tm) mode. The melting temperature was determined by calculating the first derivative from the melting curve using GraphPad Prism 5 (GraphPad Software, La Jolla, CA, United States). All measurements were performed in duplicate.

\section{Starch Synthase Activity Assays}

The activity of WT and mutated OsttaSSIII-B proteins was determined following the formation of inorganic phosphate (Pi) after hydrolysis of generated ADP by alkaline phosphatase by colorimetric assays as previously reported (Barchiesi et al., 2017). The reaction medium $(50 \mu \mathrm{l}$ final volume) contained $50 \mathrm{mM}$ BICINE pH 8.0, $2 \mathrm{U}$ of E. coli alkaline phosphatase (SigmaAldrich, MO, United States), $0-1 \mathrm{mM}$ ADPGlc and one of the following polysaccharides: $0-2 \mathrm{mg} / \mathrm{ml}$ rabbit liver glycogen (G8876 Sigma-Aldrich), 0-3 mg/ml potato AP (10118; Fluka), 0$1 \mathrm{mg} / \mathrm{ml}$ potato AM (10130; Fluka), or $0-2 \mathrm{mg} / \mathrm{ml}$ potato starch (85649; Fluka). Reaction vessels were gently shaken all over the assay to keep substrates in suspension. When UDPGlc was used as the glycosyl donor, all other assay conditions were identical, but ADPGlc was replaced with UDPGlc in the range of $0-10 \mathrm{mM}$. After incubation for $20 \mathrm{~min}$ at $30^{\circ} \mathrm{C}$, the reaction was stopped by the addition of Malachite Green-ammonium molybdate (MGam) reagent. This $M G$-am solution was prepared by merging three volumes of $0.5 \mathrm{mM}$ aqueous solution of Malachite Green (oxalate salt from Sigma, M6880) and one volume of $0.034 \mathrm{M}$ ammonium molybdate in $4 \mathrm{M} \mathrm{HCl}$. The color reaction was developed by mixing samples ( 1.0 volume) with the color reagent (6.7 volumes). The complex formed from the released Pi was measured at $630 \mathrm{~nm}$ as described (Fusari et al., 2006).

Enzyme kinetic data were plotted as specific activity $(\mu \mathrm{mol} / \mathrm{min} / \mathrm{mg}$ protein) versus substrate concentration. All kinetic parameters $\left[V_{\max }, S_{0.5}\right.$ and Hill number $\left.\left(n_{\mathrm{H}}\right)\right]$ were determined from a non-linear fit of the Michaelis-Menten equation to the observed enzyme activities using GraphPad Prism v.6.01. The average values of at least three determinations \pm SE are reported. Total protein concentration was determined using the Bradford method (Bradford, 1976).

A $\mathrm{pH}$ profile of OsttaSSIII-B activity was obtained by measuring activity at $30^{\circ} \mathrm{C}$ and $\mathrm{pH}$ values ranging from 6.0 to 10.0 
in $50 \mathrm{mM}$ BICINE buffer. The temperature profile of OsttaSSIII$\mathrm{B}$ activity was followed by measuring activity at $\mathrm{pH} 8.0$ and temperature values ranging from 20 to $45^{\circ} \mathrm{C}$ in $50 \mathrm{mM}$ BICINE buffer. Both experiments were run with $2 \mathrm{mg} / \mathrm{ml}$ glycogen and $0.5 \mathrm{mM}$ ADPGlc. Standard kinetic parameters were determined at $\mathrm{pH} 8.0$ and $30^{\circ} \mathrm{C}$.

\section{Polysaccharide-Binding Assays}

The adsorption constant $\left(K_{\mathrm{ad}}\right)$ was measured as described previously with minor modifications (Valdez et al., 2008; Wayllace et al., 2010; Barchiesi et al., 2015). Purified recombinant proteins (final concentration of $0-80 \mu \mathrm{M}$ ) were mixed with starch, AM, or AP in $20 \mathrm{mM}$ TRIS-HCl ( $\mathrm{pH} \mathrm{7.5)} \mathrm{at} \mathrm{a} \mathrm{final}$ polysaccharide concentration of $10 \%(\mathrm{w} / \mathrm{v})$ in a final volume of $60 \mu \mathrm{l}$. A control without polysaccharide was included. Binding was performed at $4^{\circ} \mathrm{C}$ for $8 \mathrm{~min}$ with gentle shaking $(10 \mathrm{rpm})$ and centrifuged at $12,000 \times g$ for $1 \mathrm{~min}$. To calculate the amount of bound protein, the total protein present in the pellet of the control reaction was subtracted from the amount of protein obtained in the pellet of the reactions with each polysaccharide. Protein concentration was determined by Bradford method (Bradford, 1976), and the adsorption constant ( $K_{\mathrm{ad}}$, in $\mathrm{ml}$ per $\mathrm{g}$ of polysaccharide) was determined from the slope as previously reported (Valdez et al., 2008; Wayllace et al., 2010; Barchiesi et al., 2015).

\section{RESULTS AND DISCUSSION}

\section{Predicted Polysaccharide-Binding Residues and Conserved Structural Features of OsttaSSIII-B}

We previously reported that $O$. tauri possesses three SSIII isoforms. Analysis of the OsttaSSIII-B amino acid sequence using the SSDB Motif Search server (Sato, 2001) revealed that this protein contains three $\mathrm{N}$-terminal SBDs $\left(E\right.$-values $=9.5 \mathrm{e}^{-6}$, $2.7 \mathrm{e}^{-12}$, and $3.9 \mathrm{e}^{-15}$, respectively; Figure 1A). Our results indicate that the domain organization of OsttaSSIII-B is most similar to the SSIII from higher plants than the other two OsttaSSIII isoforms. Figure 1B shows the amino acid sequences alignment between SBDs from OsttaSSIII-B with the following previously characterized SBDs: D1, D2, and D3 N-terminal type SBDs from ArathSSIII; a C-terminal type SBD from Aspergillus niger glucoamylase (Genbank: CAK38411.1); the glycogen-binding domain (GBD) of an AMP-activated protein from $R$. norvegicus; CBM25 from $B$. halodurans amylase; and CBM25 beta/alpha-amylase from $P$. polymyxa. These structures were used as templates for homology modeling. The starch binding sites described for ArathSSIII D2 are highly conserved in OsttaSSIII-B SBDs, specifically residues G335, W340, and Y394 (ArathSSIII numbering) belonging to binding site 1 (Valdez et al., 2011; Barchiesi et al., 2015). By contrast, binding site 2, which is essential for binding to starch, AM, and AP, is only conserved in OsttaSSIII-B D2 (W279, OsttaSSIII$\mathrm{B}$ numbering). However, the neighboring residue W365 is conserved in OsttaSSIII-B D1 (W122, OsttaSSIII-B numbering), and another aromatic amino residue is present at position 124 (Y124).

Analysis of the OsttaSSIII-B catalytic domain (CD) amino acid sequence was also performed with the SSDB Motif Search server. We found that half of the $\mathrm{CD}$ has a structure similar to the GT5 family (Pfam 343 PF08323), while the other half has a structure similar to GT1 (Pfam PF00534), with relatively low $E$-values ( $9 \mathrm{e}^{-42}$ and $2.4 \mathrm{e}^{-9}$, respectively; Figure 1A). Alignment of OsttaSSIII-B CD, OsttaSSIII-C, ArathSSIII-CD, EsccoGS, and AgrtuGS amino acid sequences revealed high conservation of residues involved in substrates binding and catalysis in the O. tauri SSIII-B isoform (Figure 1C and Tables 1, 2).

It was not possible to construct a homology model of the fulllength protein due to lack of a crystal structure of a protein with the same structural organization as SSIII, with three N-terminal SBDs and one C-terminal CD. Instead, we have built homology models of the individual SBDs (Barchiesi et al., 2015) (see Figure 2). Despite the overall architecture of each SBD being quite similar, differences in amino acid residues in the ligandbinding sites, and the relative distances between them and the catalytic domain may impose different specificities for portions of a starch molecule. These observations motivated us to probe the functional importance of this organization by comparing the activity of WT OsttaSSIII-B with mutants harboring altered starch binding sites, and a truncated OsttaSSIII-B CD variant lacking the SBDs.

\section{Expression and Purification of WT and Mutated OsttaSSIII-B Proteins}

DNA fragments encoding full-length OsttaSSIII-B and truncated OsttaSSIII-B CD were cloned into the pRSET-C vector for expression of proteins with $\mathrm{N}$-terminal His-tags. To analyze the functions of the aromatic residues W122, Y124, F138, Y147, W279, and W304 in OsttaSSIII-B polysaccharide binding and enzymatic activity, the modified proteins OsttaSSIII-B W122AY124A, F138A, Y147A, W279A, and W304A were obtained by site-directed mutagenesis. Western blot assays revealed that the size of the mutated proteins was comparable to that observed for the WT enzyme (Figure 3A), with a molecular mass of $103.2 \mathrm{kDa}$ for full-length forms and $56.3 \mathrm{kDa}$ for OsttaSSIII-B CD. Protein melting temperature curves were obtained for WT OsttaSSIII-B, the truncated $\mathrm{CD}$ variant, and five modified enzymes, and the melting temperature (Tm) was determined (Figures 3B,C). Fluorescencebased thermal shift assays showed that the melting profiles of mutant proteins were similar to that of the WT enzyme, indicating that overall folding was conserved. Nevertheless, the mutation F138A caused a decrease of $10^{\circ} \mathrm{C}$ in $\mathrm{Tm}$, whereas mutations W122AY124A and Y147 lowered the Tm by 2.5 and $4.5^{\circ} \mathrm{C}$, respectively. OsttaSSIII-B CD showed two distinct melting temperatures, both higher than the Tm value for the full-length WT enzyme $\left(46.2\right.$ and $\left.57.5^{\circ} \mathrm{C}\right)$. This observation may suggest successive melting of independent protein domains caused by the absence of the N-terminal region. From these results, we conclude that the mutated 
A

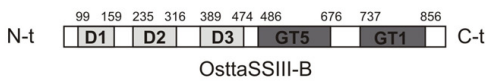

B

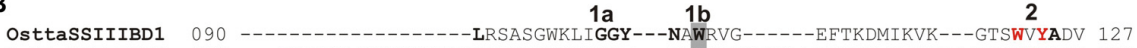
OsttaSSIIIBD2 224 ---GSTERV-MWNKAYNPIGGDTDKLVLHVGF---NGWCAGVEAK-FDLVRATGEIKDE-NNDWWVADV 283 OsttaSSIIIBD3 385 ---GKPLKV-FYNKN-NTNLSWTDEIYMTGGF---NRWSHEA----AIEPVKMTPPGAG-EEFFTVTVP 440 ArathSSIII D1 134 -KPDEDTEV-FT NRN-LSTINNEPDVLIMGAF---NEWRWKS------FTRRTEKTWTHE--DWLSCLL 188 ArathSSIII D2 309 -KAEDTVKL-YYNKR-SGPLTNSKELWLHGG---NNWVDGLSIVVKIVNARLKDVDPR-SGNWWFAEV 370 ArathSSIII D3 476 - -AAGPVVTV-YYNPA-NTVINGKPEVWFGGSF---NRWTHRLG--DPPPOKMEATDD--ESSHVKTTA 533

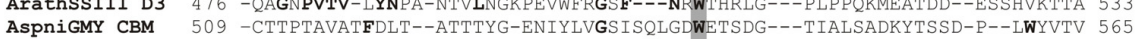
PaepoAMY CBM 455 GGT GNKVTI I-YYKKGFNS-------PYIHYRPAGGSWTAAP----G-VKMQDAEIS-----GYAKITV 504 BachaAMY CBM 006 SGDATDITI-YYKTGWTH-------PHIHYSINQGAWTTLP----G-VPLTKS---------VKVTI 050 RatnoAMPK GBD 079 -----PTVERWTGGGKE-----------VYLSGSENNWS-K-------LPLTRSQ-------NNFVAIL 116

\begin{tabular}{|c|c|c|c|}
\hline IIIBI & 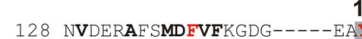 & & \\
\hline OsttaSSIIIBD2 & 284 PLRADAYLLDFVVSDVEE-S & & \\
\hline OsttaSSIIIBD3 & 441 RVPTDANMVDFVF SSGVG-EG & QYDNKSGRDYHLP- & 474 \\
\hline athSSIII D1 & 189 & :KDFCVEIKGGMD & \\
\hline & 371. & & \\
\hline thSSIII D3 & 534 KVPLI & & \\
\hline oniGMY CBM & TLP-AGESFEYKFTRTE & NRE YTVPC & -616 \\
\hline epoAMY CBM & & NSGNAGT & \\
\hline achaAMY CBM & EEGS-OLRAAFNNGSG- & & \\
\hline EnOAMPI & & & \\
\hline
\end{tabular}

C

OsttaSSIIIB CD 475 -TRGSTASK----PPLHVCHIAVEMAPIARVGGLA3 4903 DVVTAIGRAIQDNGHLVEI ILPKYQFFNNSVLLGGK 540

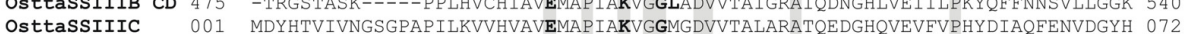

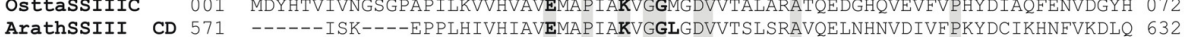
ArathSSIII CD 571 ------ISK----EPPLHIVHIAVEMAP IAKVGGLGDVVTSLSRAVQELNHINVDIVF PKYDCIKHNFVKDLQ 632

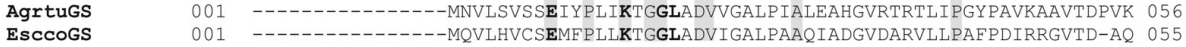

OsttaSSIIIB CD 541 EYETHFDWAGTTIRVEKCKVEGLQCFFIEPQNNMFQTDSVYG------RNDDAHRFNFFCNAALEFLV--RT 604 OsttaSSIIIC 073 -RAGEFKHEKTVVQVYKGWVEDVPVTLLLRPENGFFDVGCIYG------RGDDHVRFDFFTDATLTWLR--SK 137 ArathSSIII CD 634 -FNRSYHWGGTEIKVWHGKVEGLSVYFLDPQNGLFQRGCVYG------CADDAGRFGFFCHAALEFLL--QG 695 AgrtuGS 057 CFEFTDLLGEKADLLEV-QHERLDLLILDAPAYYERSGGPYLGQTGKDYPDNWRRFAALSLAAARIGAGVLP 127 EsccoGS 056 VVSRRDTFAGHITLIFG-HYNGVGIYLIDAPHLYDRPGSPYHDTNLFAYTDNVLRFALLGWVGAEMASGLDP 126

OsttaSSIIIB CD 605 ARQPDILHC 615

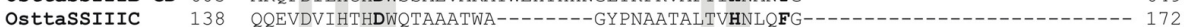

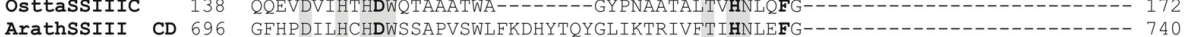

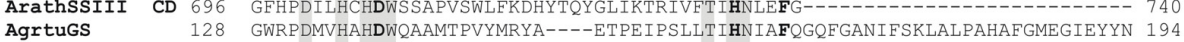
EscCoGS 127 FWRPDVVHAHDWHAGLAPAYLAR----GRPAK-SVETVHNLAYQGMFYAHHMNDIQLPWSFFNIHGLEFNG 192

OsttaSSIIIB CD 650 -QAKIGEAVHHSQVATTVSPSYAGEVRGSPVIG-------NNGHKETGVRNGIDPEIWDPETDIFVPVKY 711 OsttaSSIIIC 173 -VDRIRRGMESCDIATTVSPTYADEVRFHHAIA---------PSKDKFIGIRNGIDTDIWNPANDKFLPVGY 234 ArathSSIII CD 741 -ANAIGKAMTFADKATTVSPTYAKEVAGNSVISAHL---------YKFHGI INGIDPDIWDPYNDNFI PVPY 802 AgrtuGS 195 DVSFLKGGLOTATALSTVSPSYAEEILTAEFGMGLEGVIGS--RAHVLHGIVNGI DADVWNPATDHL.T HDNY 266 EscCOGS 193 OISFLKAGLYYADHITAVSPTYREITEPOFAYGMEGLIOORHREGRLSGVLNGVDEKIWSETDLLLASRY 266

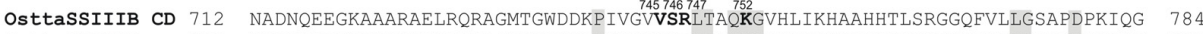
OsttaSSIIIC 235 NRSNAIDGKRAAAAELCNRLGLEHPEGSPIVGVVSRLTAQKGIHLIKHACYRVLERGATFVLLGNAPDPAHQH 307 ArathSSIII CD 803 TSENVVEGKRAAKEE LQNRLGLKS-ADFPVVGI ITRLTHQKGIHLIKHAIWRTLERNGQVVLLGSAPDPRIQN 874 AgrtuGS 256 SAAN-LKNRALNKKAVAEHFRID-DDGSPLFCVISRLTWQKGIDLMAEAVDEIVSIGGRLVVLG-AGDVALEG 339 EsccoGS 256 TRDT-LEDKAENKRQLQIAMGLKVDDKVPLFAVVSRLTSQKGLDLVLEALPGLLEQGGQLALLG-AGDPVLQE 340

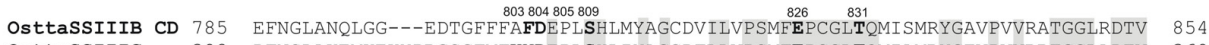
OsttaSSIIC 308 DFNSLAKEMKEKYPRGSGFMFKYDEPLSHLIYAGCDELIVPSMFEPCGLTQMIAMRYGTVPVVRRTGGLADTV 369 $\begin{array}{llll}\text { OsttaSSIIC } & 308 & \text { DFNSLAKEMKEKYRGSGFMF KYDEPLSHLIYAGCDFLIVPSMFE PCGLTQMIAMRYGTVVVRRTGGLADTV } & 369 \\ \text { ArathSSIII CD } & 875 & \text { DFVNLANQLHSSGDRARLVLTYDEPLSHLIYAGADILVPSIFEPCLTQLIAMRYGAVPVVRKTGGLFTV } & 947\end{array}$ $\begin{array}{lllll}\text { ArathSSIII CD } & 875 & \text { DFVNLANQLHSSHGDRARLVLTYDEPLSHLIYAGADFILVPSIFEPCGLTQLIAMRYGAVPVRKTGGLFDTV } & 947 \\ \text { AgrtuGS } & 340 & \text { ALIAAASRHHG----RVGVAIGYNEPLSHLMOAGCDAIII PSRFEPCGLTOLYALRYGCI PVVARTGGLADTV } & 397\end{array}$

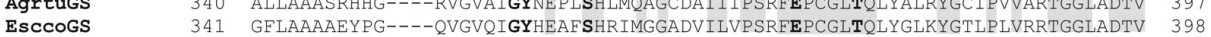

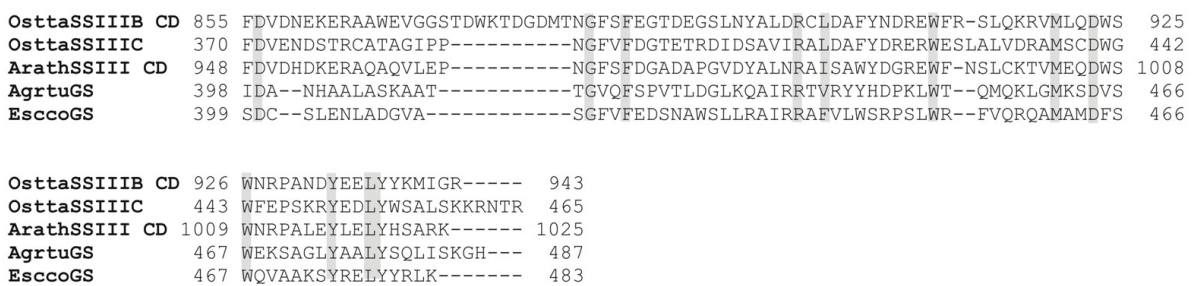

FIGURE 1 | (A) Schematic representation of OsttaSSIII-B. D1, D2, D3, GT5, and GT1 domains are shown. (B) ClustalW alignment of OsttaSSIII-B D1, D2, and D3, ArathSSIII (Arabidopsis thaliana SSIII) D1, D2, and D3, AspniGMY (A. niger glucoamilase) CBM20, and RatnoAMPK $\beta$-subunit GBD (CBM48), BachaAMY CBM25, and PaepoAMY CBM25, used as templates for starch-binding domain (SBD) homology modeling. 1 and 2 above the alignment indicate binding sites 1 and 2 characterized in A. thaliana. 1a, 1b, and 1c indicate G335, W340, and Y394, and 2 indicates W366 (ArathSSIII numbering). OsttaSSIII-B amino acids residues W122, Y124, F138, Y147, W279, and W304 are indicated in red. Other previously characterized binding residues are shown in bold. Gray boxes indicate conserved residues. (C) ClustalW alignment of OsttaSSIII-B, OsttaSSIII-C, ArathSSIII-CD (Arabidopsis thaliana SSIII catalytic domain), AgrtuGS (Agrobacterium tumefaciens glycogen synthase), and EsccoGS (E. coli glycogen synthase). Previously characterized catalytic residues are shown in bold. Gray boxes indicate identical residues. 
TABLE 1 | Amino acid residues involved in glycogen binding in ArathSSIII CD, EsccoGS, and OsttaSSIII-B CD.

\begin{tabular}{llll}
\hline EsccoGs & OsttassIII-B & ArathSSIII & Function \\
\hline Glu9 & Glu493 & Glu585 & Binding of Glc +1 \\
Leu19 & Leu503 & Leu595 & Binding of Glc +1 (KTGGL/KVGGM) \\
Tyr 95 & Tyr581 & Tyr672 & Stacking With Glc +2 \\
Asp137 & Asp615 & Asp706 & H-bond with $\mathrm{OH}_{2}$ and $\mathrm{OH}_{3}$ Glc +1 \\
Tyr165 & Tyr648 & Phe740 & Stacking with Glc +2 \\
\hline
\end{tabular}

Ref: For the Arabidopsis thaliana SSIII isoform, Glc +1 and Glc +2 indicate which $\mathrm{Glc}$ in glycogen is involved in the interaction; $\mathrm{OH} 2$ and $\mathrm{OH} 3$ indicate the $\mathrm{OH}$ groups of Glc +1 that interact with the protein; KTGGL and KVGGM correspond to the conserved motifs in E. coli GS and OsttaSSIII-B enzymes, respectively.

TABLE 2 | Amino acid residues involved in ADPGlc binding in ArathSSIII, EsccoGS, and OsttaSSIII-B.

\begin{tabular}{llll}
\hline EsccoGs & OsttaSSIII-B & ArathSSIII & Function \\
\hline Lys15 & Lys499 & Lys591 & ADPGlc binding (KTGGM/KVGGL) \\
Gly18 & Gly502 & Gly594 & ADPGlc binding (KTGGM/KVGGL) \\
His161 & His644 & His736 & H-bond with O6 ribose (380 loop) \\
Asn246 & Asn692 & Asn783 & H-bond with $\mathrm{O}_{6}$ ribose \\
Val297 & Val745 & Ile835 & H-bond with $\mathrm{O}_{2}$ ribose \\
Ser298 & Ser746 & Thr836 & vdW with adenine \\
Arg300 & Arg747 & Arg837 & lonic interaction with Pi \\
Lys305 & Lys752 & Lys842 & Ionic interaction with Pi \\
Gly354 & Ala803 & Thr896 & Interaction with adenine \\
Tyr355 & Phe804 & Tyr897 & Stacking with adenine \\
His356 & Asp805 & Asp898 & Carbonyl interacts with adenine \\
Ser360 & Ser809 & Ser902 & vdW with adenine \\
Glu377 & Glu826 & Glu919 & lonic interaction with Pi \\
Thr382 & Thr831 & Thr924 & H-bond with O 2 ribose \\
\hline
\end{tabular}

Ref: AthSSIII, Arabidopsis thaliana SSIII isoform; Adenine, ribose and phosphate (Pi) indicate which part of the ADP-GIc participates in each interaction; O2 and O6, oxygen atoms in glucose; vdW, van der Waals interactions; KTGGM and KVGGL correspond to the conserved motifs in E. coli GS and OsttaSSIII-B enzymes, respectively.

proteins were properly folded, but their thermal stability was somewhat reduced by introduction of mutations in the D1 domain.

\section{Kinetic Characterization of OsttaSSIII-B}

Kinetic parameters were calculated for WT OsttaSSIII-B from saturation plots of enzymatic activity using ADPGlc as the sugar-nucleotide donor, and glycogen, starch, AM, or AP as the acceptor substrate (Figures $4 \mathrm{~A}, \mathbf{5 A}, \mathbf{B}$ and Table 3). In all conditions, OsttaSSIII-B exhibited typical Michaelis-Menten kinetics. The $S_{0.5}$ values for the acceptor polysaccharide were $0.08 \mathrm{mg} / \mathrm{ml}$ for glycogen, $0.12 \mathrm{mg} / \mathrm{ml}$ for starch, $0.08 \mathrm{mg} / \mathrm{ml}$ for $\mathrm{AM}$, and $1.04 \mathrm{mg} / \mathrm{ml}$ for AP. By contrast, the $S_{0.5}$ value for ADPGlc was $90 \mu \mathrm{M}$ when glycogen was used as the acceptor substrate (Table 3). The $S_{0.5}$ value for glycogen was about $75 \%$ lower than those reported for other SSIIIs, such as OsttaSSIII-C and ArathSSIII $\left(S_{0.5}=0.23\right.$ and $0.26 \mathrm{mg} / \mathrm{ml}$, respectively) (Valdez et al., 2008; Wayllace et al., 2010; Barchiesi et al., 2017). On the other hand, $n_{\mathrm{H}}$ values for ADPGlc and glycogen were about 1.16 and 0.98 , respectively, whereas for AP and AM were 1.48 and 1.60 , respectively, suggesting a cooperative kinetics for these polysaccharides.

OsttaSSIII-B specific activity was higher with glycogen respect to those obtained for starch, AM, or AP (80-, 537-, and 5-fold higher, respectively). The lower specific activity observed with starch may be due to an inhibitory effect of the amylose present in this polysaccharide. It should be noted that OsttaSSIII-B showed a lower $S_{0.5}$ for amylose than for starch or amylopectin (see Table 3).

Similar results, demonstrating highest activity with glycogen as acceptor substrate, were previously reported for ArathSSIII (Valdez et al., 2008) and OsttaSSIII-C (Barchiesi et al., 2017). OsttaSSIII-B did not exhibit enzymatic activity under the assayed conditions when UDPGlc was used as the donor substrate. Furthermore, OsttaSSIII-B displayed an optimum reaction temperature of $30^{\circ} \mathrm{C}$, and a $\mathrm{pH}$ optimum of 8 (Figures $4 \mathbf{B}, \mathbf{C}$ ), consistent with the alkaline $\mathrm{pH}$ of the chloroplast stroma. Based on these results, we further studied the kinetic parameters of OsttaSSIII-B WT, truncated, and modified forms using glycogen as the acceptor polysaccharide and ADPGlc as the glycosyl donor, at $\mathrm{pH} 8$ and $30^{\circ} \mathrm{C}$.
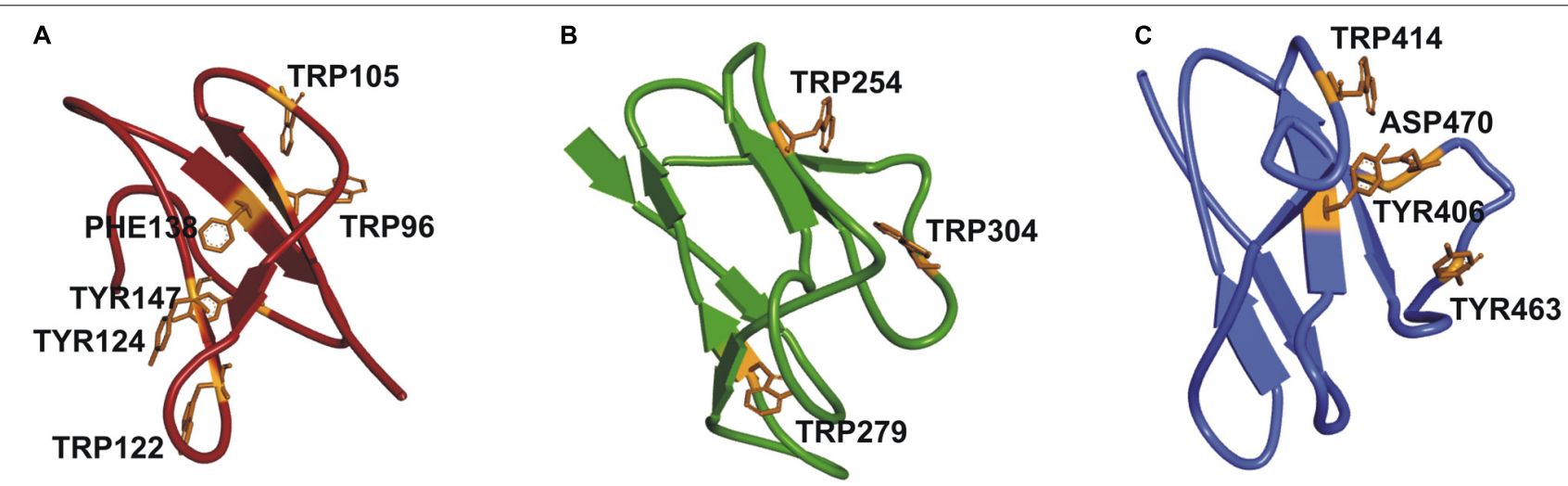

FIGURE 2 | Homology modeling of OsttaSSIII-B SBDs (A) D1 (red); (B) D2 (green), and (C) D3 (blue) domains, respectively, of OsttaSSIII-B, showing the residues involved in binding in orange. 


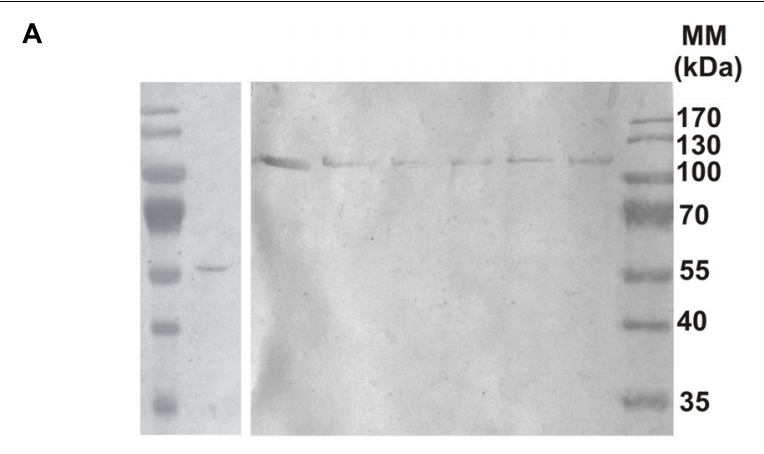

B

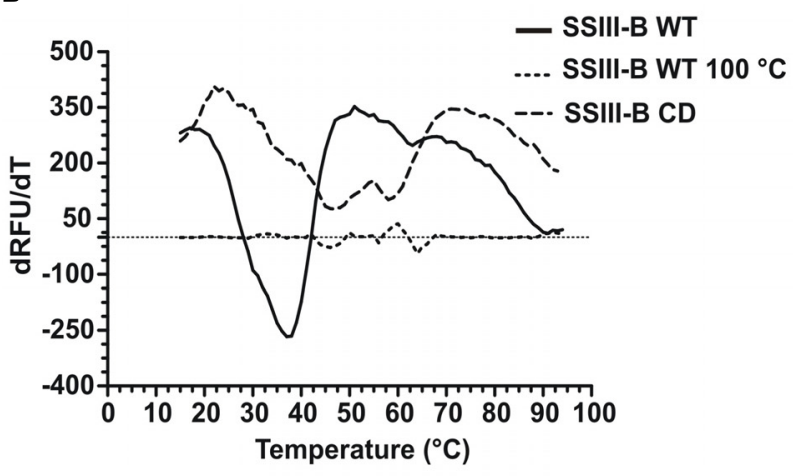

C

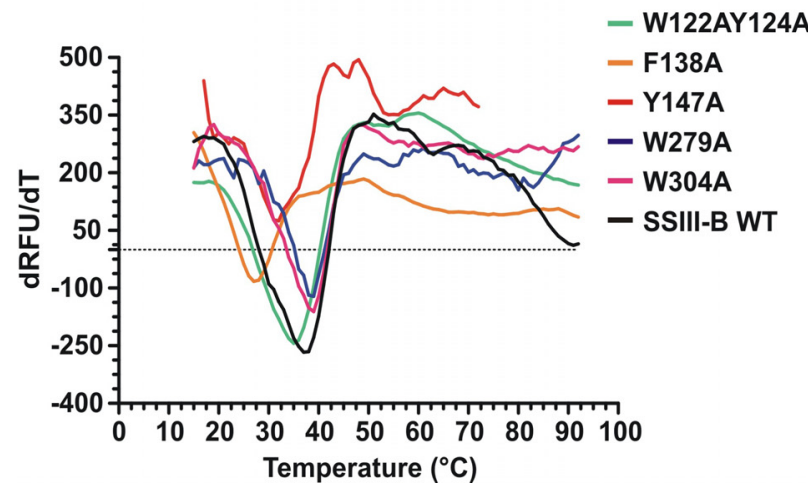

FIGURE 3 | (A) Western blot of recombinant enzymes. Lane 1, Molecular weight standards; lane 2, OsttaSSIII-B CD; lane 3, OsttaSSIII-B WT; lane 4, OsttaSSIII-B W122AY124A; lane 5, OsttaSSIII-B F138A; lane 6, OsttaSSIII-B Y147A; lane 7, OsttaSSIII-B W279A; lane 8, OsttaSSIII-B W304A. Numerals indicate molecular masses of the prestained SDS-PAGE standards (Low Range, BioRad). (B,C) First derivatives $[-\mathrm{d}(\mathrm{RFU}) / \mathrm{dT}]$ from the melting curves determined using differential scanning fluorimetry. Melting temperature (Tm) was obtained from the $x$-axis value (T) that corresponds with the lowest $y$-axis value of the curve. SSIII-B $100^{\circ} \mathrm{C}$ is the curve corresponding to the wild-type (WT) protein previously denatured by heating for $15 \mathrm{~min}$ at $100^{\circ} \mathrm{C}$, used as an unfolded protein control.

\section{The N-Terminal SBDs Are Important for OsttaSSIII-B Starch Synthase Activity}

In order to analyze the role of the SBDs in the function of OsttaSSIII-B, we evaluated the starch synthase activity of the truncated OsttaSSIII-B CD protein (Figures 5B,C and Tables 4, 5). Under the assay conditions, OsttaSSIII CD exhibited a $50 \%$ lower $V_{\max }$ value than that of the full-length enzyme, whereas the
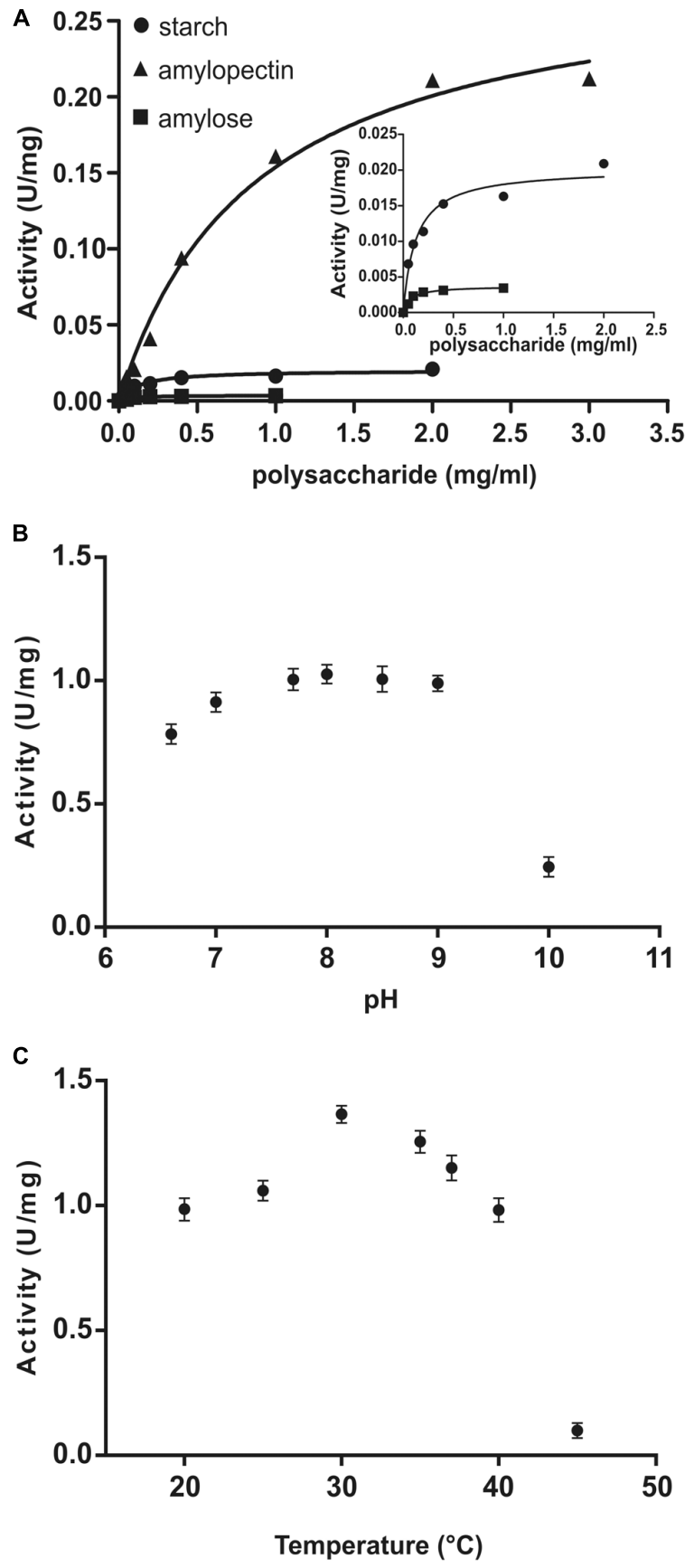

FIGURE 4 | (A) Starch (circles), amylose (squares), and amylopectin (triangles) saturation plots for WT OsttaSSIII-B measured in the presence of $500 \mu \mathrm{M}$ ADPGlc. (B) Effect of $\mathrm{pH}$ on the activity of WT OsttaSSIII-B. $50 \mathrm{mM}$ BICINE and $50 \mathrm{mM}$ glycine buffers were used for $\mathrm{pH} 6.0$ - 10.0. Assays were run with $2 \mathrm{mg} / \mathrm{ml}$ glycogen and $500 \mu \mathrm{M}$ ADPGlc. (C) Effect of temperature on the activity of WT OsttaSSIII-B. Assays were run with $2 \mathrm{mg} / \mathrm{ml}$ glycogen and $500 \mu \mathrm{M}$ ADPGlc, in $50 \mathrm{mM}$ BICINE buffer $\mathrm{pH}$ 8, with reaction temperatures of $20,25,30,35,40$, and $45^{\circ} \mathrm{C}$.

$S_{0.5}$ value for ADPGlc was similar. The $S_{0.5}$ value for the acceptor polysaccharide was twofold higher than the full-length protein $(0.18$ and $0.08 \mathrm{mg} / \mathrm{ml}$, respectively; Tables 4,5$)$. These results 
TABLE 3 | Kinetic parameters of wild-type (WT) OsttaSSIII-B.

\begin{tabular}{llcr}
\hline & $\begin{array}{l}\boldsymbol{S}_{\mathbf{0 . 5}} \text { (ADPGlc } \mathbf{~ m M} \\
\text { Polysacc } \mathbf{~ m g / m l )}\end{array}$ & $\boldsymbol{n}_{\mathbf{H}}$ & $\boldsymbol{V}_{\mathbf{m a x}}(\mathbf{U} / \mathbf{m g})$ \\
\hline ADPGlc & $0.09 \pm 0.02$ & $1.16 \pm 0.23$ & $1.65 \pm 0.09$ \\
Glycogen & $0.08 \pm 0.02$ & $0.98 \pm 0.10$ & $1.61 \pm 0.09$ \\
Starch & $0.12 \pm 0.02$ & $0.48 \pm 0.20$ & $0.02 \pm 0.01$ \\
Amylopectin & $1.04 \pm 0.17$ & $1.48 \pm 0.23$ & $0.30 \pm 0.02$ \\
Amylose & $0.08 \pm 0.01$ & $1.60 \pm 0.22$ & $0.003 \pm 0.001$ \\
\hline
\end{tabular}

suggest that the presence of the SBDs correlates with an increase in the apparent affinity for glycogen.

Amino acid sequence alignment and homology modeling revealed the high conservation of specific residues in SBDs potentially implicated in starch binding and catalysis. We previously demonstrated that the OsttaSIII-B D1 and D2 domains share higher sequence identity with ArathSSIII D2 than OsttaSIII-B D3, and that both domains make major contributions to polysaccharide binding. The putative starch-binding residues in the OsttaSIII-B D2 domain, as well as the shorter OsttaSIIIB D1 domain (60 amino acid residues), are highly conserved in ArathSSIII D2 (Barchiesi et al., 2015).

The amino acid residues Y147 and W304 of OsttaSSIII-B align well with Y394 of the ArathSSIII D2 1c binding site, and W279 aligns with W366 of the ArathSSIII D2 binding site 2 (Figure 1A). Additionally, we previously postulated that amino acids W122 and Y124 of OsttaSSIII-B belong to binding site 2 in D1. However, residue F138 is also highly conserved, but this residue has not been characterized before. Therefore, to analyze the functions of these amino acid residues in OsttaSSIII-B activity, kinetic parameters of alanine-substituted mutants were determined as described above in the Materials and Methods section, and the results are shown in Figure 5 and Tables 4, 5. An approximately $50-90 \%$ decrease in $V_{\max }$ was observed for
TABLE 4 | Kinetic parameters of WT SSIII-B and mutated proteins for glycogen.

\begin{tabular}{lcccc}
\hline Isoform & $\boldsymbol{S}_{\mathbf{0 . 5}} \mathbf{( m g / m l )}$ & $\boldsymbol{n}_{\mathbf{H}}$ & $\boldsymbol{V}_{\max }(\mathbf{U} / \mathbf{m g})$ & $\boldsymbol{V}_{\mathbf{m a x}} / \mathbf{S}_{\mathbf{0 . 5}}$ \\
\hline SSIII-B WT & $0.08 \pm 0.02$ & $0.98 \pm 0.10$ & $1.61 \pm 0.09$ & $20.12 \pm 0.30$ \\
SSIII-B & $0.31 \pm 0.09$ & $1.80 \pm 0.23$ & $0.71 \pm 0.06$ & $2.29 \pm 0.37$ \\
W122A-Y124A (D1) & & & & \\
SSIII-B F138A (D1) & $0.40 \pm 0.06$ & $0.84 \pm 0.16$ & $0.28 \pm 0.01$ & $0.70 \pm 0.18$ \\
SSIII-B Y147A (D1) & $0.25 \pm 0.05$ & $1.02 \pm 0.11$ & $1.24 \pm 0.07$ & $4.96 \pm 0.26$ \\
SSIII-B W279A (D2) & $0.54 \pm 0.08$ & $0.93 \pm 0.09$ & $0.14 \pm 0.01$ & $0.26 \pm 0.22$ \\
SSIII-B W304A (D2) & $0.89 \pm 0.20$ & $0.88 \pm 0.10$ & $0.19 \pm 0.02$ & $0.21 \pm 0.33$ \\
SSIII-B CD & $0.18 \pm 0.10$ & $2.00 \pm 1.32$ & $0.78 \pm 0.12$ & $4.33 \pm 0.71$
\end{tabular}

the mutated proteins compared with the WT enzyme, except for the Y147A variant. Using glycogen as the variable substrate, $S_{0.5}$ values for the polysaccharide were approximately 3 - to 10 -fold higher respect to that observed for the WT enzyme $(0.31,0.40$, $0.25,0.54$, and $0,89 \mathrm{mg} / \mathrm{ml}$ for W122AY124A, F138A, Y147A, W279A, and W304A, respectively) (Table 4 and Figure 5). These results show that there is a remarkable decrease in the apparent affinity for the polysaccharide substrate, while minor changes (until fourfold) in the $S_{0.5}$ for ADPGlc were observed (Table 5).

It is important to note that the $S_{0.5}$ for glycogen of the CD protein is lower than that of any of the mutated enzymes. While the SBDs present in the full length WT protein increases the affinity for glycogen, the mutated inactive SBDs could block the access of the substrate to the catalytic site, resulting in higher $S_{0.5}$ values.

\section{Effect of SBD Amino Acid Substitutions on OsttaSSIII-B Binding Properties}

We next explored the effect of amino acid substitutions in SBD regions on substrate binding. Figure 6 shows adsorption

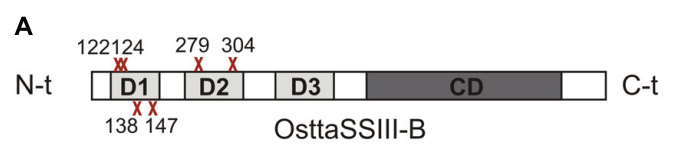

B

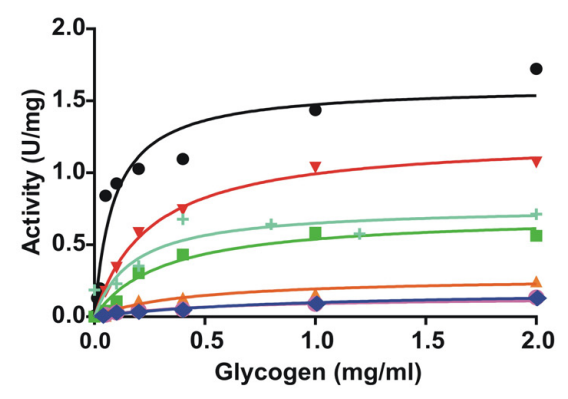

C

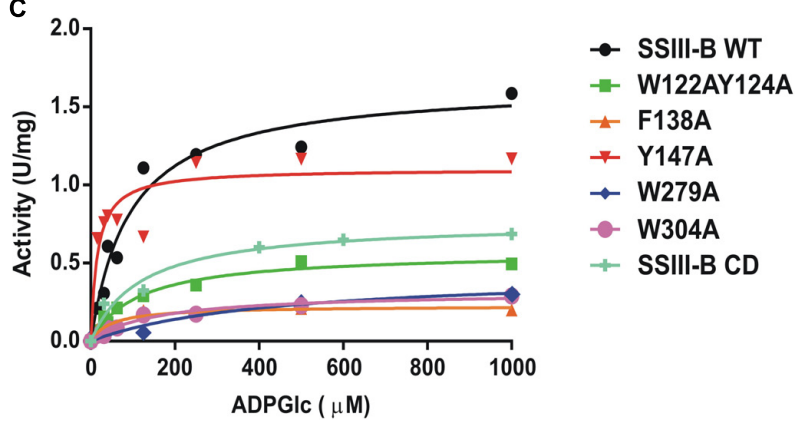

FIGURE 5 | (A) Schematic representation of the mutated proteins used in this study. Residues W122, Y124, F138, Y147, W279, and W304 were replaced by alanine to yield OsttaSSIII-B W122AY124A, F138A, Y147A, W279A, and W304A. Amino acid locations are indicated with a red cross. CD, catalytic domain; D1, D2, and D3, individual SBD modules. (B) Glycogen and (C) ADPGlc saturation plots for WT OsttaSSIII-B and mutated enzymes performed in the presence of $500 \mu \mathrm{M}$ ADPGlc or $2 \mathrm{mg} / \mathrm{ml}$ glycogen, respectively. 
TABLE 5 | Kinetic parameters of SSIII-B WT and mutated proteins with the substrate ADPGlc.

\begin{tabular}{lcccc}
\hline Isoform & $\boldsymbol{S}_{\mathbf{0 . 5}}(\mathbf{m M})$ & $\boldsymbol{n}_{\mathbf{H}}$ & $\boldsymbol{V}_{\max }(\mathbf{U} / \mathbf{m g})$ & $\boldsymbol{V}_{\max } / \mathbf{S}_{\mathbf{0 . 5}}$ \\
\hline SSIII-B WT & $0.09 \pm 0.02$ & $1.16 \pm 0.23$ & $1.65 \pm 0.09$ & $18.33 \pm 0.27$ \\
SSIII-B & $0.11 \pm 0.01$ & $0.94 \pm 0.13$ & $0.57 \pm 0.02$ & $5.18 \pm 0.12$ \\
W122AY124A (D1) & & & & \\
SSIII-B F138A (D1) & $0.07 \pm 0.02$ & $1.06 \pm 0.33$ & $0.23 \pm 0.02$ & $3.28 \pm 0.37$ \\
SSIII-B Y147A (D1) & $0.07 \pm 0.01$ & $0.78 \pm 0.18$ & $1.10 \pm 0.08$ & $15.71 \pm 0.21$ \\
SSIII-B W279A (D2) & $0.38 \pm 0.11$ & $0.88 \pm 0.23$ & $0.42 \pm 0.05$ & $1.10 \pm 0.41$ \\
SSIII-B W304A (D2) & $0.15 \pm 0.03$ & $0.80 \pm 0.16$ & $0.31 \pm 0.02$ & $2.06 \pm 0.26$ \\
SSIII-B CD & $0.12 \pm 0.04$ & $0.57 \pm 0.28$ & $0.76 \pm 0.06$ & $6.33 \pm 0.4$ \\
\hline
\end{tabular}

TABLE 6 | Adsorption constants for OsttaSSIII-B proteins.

\begin{tabular}{lccc}
\hline Isoform & \multicolumn{3}{c}{$\boldsymbol{K}_{\text {ad }}(\mathbf{m l} \mathbf{g})$} \\
\cline { 2 - 4 } & Starch & Amylose & Amylopectin \\
\hline OsttaSSIII-B WT & $2.40 \pm 0.21$ & $16.61 \pm 0.91$ & $1.83 \pm 0.31$ \\
OsttaSSIII-B W122AY124A (D1) & $\mathrm{ND}$ & $2.44 \pm 0.82$ & $\mathrm{ND}$ \\
OsttaSSIII-B F138A (D1) & $\mathrm{ND}$ & $11.48 \pm 0.61$ & $\mathrm{ND}$ \\
OsttaSSIII-B Y147A (D1) & $2.50 \pm 0.43$ & $10.53 \pm 0.42$ & $\mathrm{ND}$ \\
OsttaSSIII-B W279A (D2) & $\mathrm{ND}$ & $\mathrm{ND}$ & $4.75 \pm 0.28$ \\
OsttaSSIII-B W304A (D2) & $\mathrm{ND}$ & $\mathrm{ND}$ & $2.75 \pm 0.26$ \\
OsttaSSIII-B CD & $\mathrm{ND}$ & $3.38 \pm 0.73$ & $\mathrm{ND}$ \\
\hline
\end{tabular}

ND, No detected.

isotherms for the binding of WT, CD, and mutant OsttaSSIIIB proteins to starch, AM, and AP (see also Table 6). WT OsttaSSIII-B displayed higher affinity for AM $(16.61 \mathrm{ml} / \mathrm{g})$ than starch $(2.40 \mathrm{ml} / \mathrm{g})$ or AP $(1.83 \mathrm{ml} / \mathrm{g})$. These results are consistent with our previous studies on binding using $\mathrm{N}$-terminal SBDs (D123) of WT SSIII-B $\left(K_{\mathrm{ad}}=2.22,7.02\right.$, and $3.84 \mathrm{ml} / \mathrm{g}$ for starch, AM, and AP, respectively) (Barchiesi et al., 2015), and also with binding of D123 from ArathSSIII (Valdez et al., 2011). The higher binding affinity observed for AM could be due to the insignificant branching degree of this polysaccharide, regards to starch or amylopectin, which could make it more accessible for the large OsttaSSIII-B protein. In the case of the CD, we verified its ability to bind AM $\left(K_{\mathrm{ad}}=3.38 \mathrm{ml} / \mathrm{g}\right)$ but the absence of SBDs could cause the lost of the binding capacity to starch and AP (Table 6). Meanwhile, W122AY124A, F138A, and Y147A variants yielded $K_{\mathrm{ad}}$ values for AM that were 7-, 1.5-, and 1.5fold lower than the WT enzyme, and all three mutants completely lost their capacity to bind AP, suggesting that these amino acid residues in $\mathrm{D} 1$ are essential for binding to the $\mathrm{AP}$ component. By contrast, W279A and W304A proteins were unable to bind AM and starch, suggesting both residues are essential for binding to the AM fraction. The Y147A protein was the only isoform capable of binding starch, suggesting that all other studied residues (W122, Y124, F138, W279, and W304) are essential for binding to physiological substrates. Analysis of the spatial position of Y147 in our OsttaSSIII-B model shows that this residue is far away from the other two conserved binding amino acids in D1 (W96 and W105), suggesting that Y147, which aligns with binding site $1 \mathrm{c}$ in the D1 domain, is not involved in starch
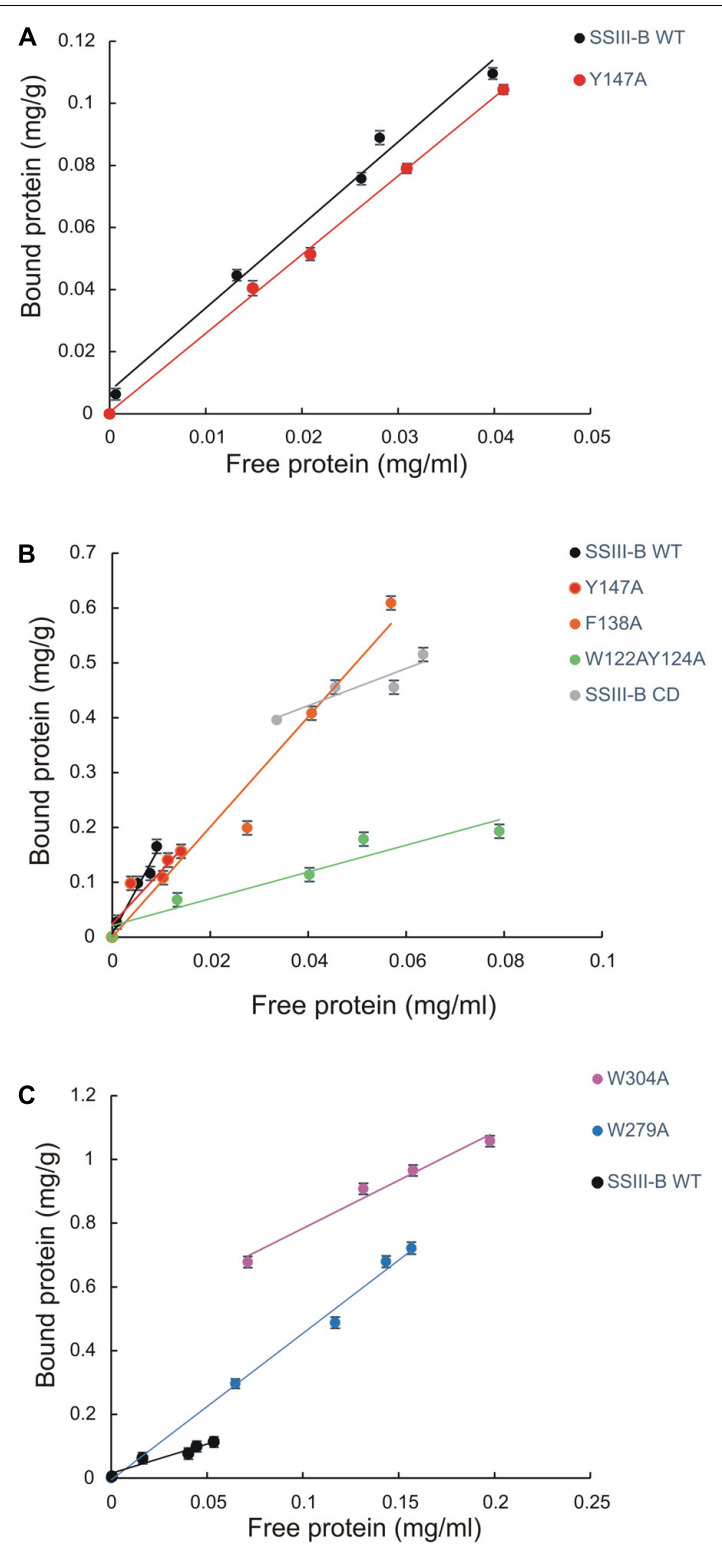

FIGURE 6 | Adsorption of purified OsttaSSIII proteins to polysaccharides. Absorption isotherms are shown with (A) starch, (B) amylose, (C) amylopectin. Linear adsorption isotherms indicate the apparent equilibrium distribution of WT OsttaSSIII-B and mutated proteins between the solid (bound protein) and liquid (free protein) phase at different protein concentrations. Adsorption constants are listed in Table $\mathbf{6}$ and represent the slopes of the isotherms with units of $\mathrm{ml}$ of protein per milligram of polysaccharide ( $n=2 \pm$ standard error).

binding, and that D1 likely utilizes another aromatic residue in this position. Interestingly, some OsttaSSIII-B-mutated proteins displayed an increased polysaccharide binding capacity relative to the WT enzyme. The $K_{\mathrm{ad}}$ values for AP of W279A and W304A variants were 2.6- and 1.5-fold higher than those observed for the WT protein $\left(K_{\mathrm{ad}} \mathrm{W} 279 \mathrm{~A}=4.75 \mathrm{ml} / \mathrm{g}\right.$, and $K_{\mathrm{ad}}$ $\mathrm{W} 304 \mathrm{~A}=2.75 \mathrm{ml} / \mathrm{g}$, vs. $\left.K_{\mathrm{ad}} \mathrm{WT}=1.83 \mathrm{ml} / \mathrm{g}\right)($ Figure $6 \mathrm{C}$ and Table 6). 
Taken together, these results suggest that the AM specificity of the WT enzyme can be attributed to the binding residues in the D2 domain, while the ability to bind AP is related to the D1 domain. However, it is important to emphasize that amino acid residues in both $\mathrm{D} 1$ and D2 are essential for binding to starch. This is the first experimental evidence demonstrating the importance of amino acid residues in the D1 domain in the binding capacity of a SS enzyme.

\section{CONCLUSION}

A better understanding of the properties of CBMs and SBDs and their interactions with catalytic domains could provide new strategies to develop enzymes with improved catalytic efficiency toward polysaccharide substrates. In previous work, we evaluated the effects of mutating several residues in the D2 domain of SSIII from A. thaliana. Herein, we analyzed the contribution of several residues from D1 and D2 domains, representing the first characterization of the effects of both domains on the entire protein.

The adsorption parameters of the full-length protein for AM were twice higher than that obtained for the D123 domain alone (Barchiesi et al., 2015), indicating an important contribution of the CD in providing additional binding sites for the linear substrate. Furthermore, mutation of the D1 domain mainly affected AP binding, whereas mutation of D2 mainly affected AM binding, indicating that both SBDs possess different capabilities in the adsorption of different substrates. In addition, mutation of the D2 domain affected the enzyme catalysis, whereas altering the D1 domain had less impact.

\section{REFERENCES}

Ball, S. G., and Morell, M. K. (2003). From bacterial glycogen to starch: understanding the biogenesis of the plant starch granule. Annu. Rev. Plant Biol. 54, 207-233. doi: 10.1146/annurev.arplant.54.031902.134927

Barchiesi, J., Hedin, N., Gomez-Casati, D. F., Ballicora, M. A., and Busi, M. V. (2015). Functional demonstrations of starch binding domains present in Ostreococcus tauri starch synthases isoforms. BMC Res. Notes 8:613. doi: 10.1186/s13104-015-1598-6

Barchiesi, J., Hedin, N., Iglesias, A. A., Gomez-Casati, D. F., Ballicora, M. A., and Busi, M. V. (2017). Identification of a novel starch synthase III from the picoalgae Ostreococcus tauri. Biochimie 133, 37-44. doi: 10.1016/j.biochi.2016. 12.003

Boraston, A. B., Bolam, D. N., Gilbert, H. J., and Davies, G. J. (2004). Carbohydratebinding modules: fine-tuning polysaccharide recognition. Biochem. J. 382, 769-781. doi: 10.1042/BJ20040892

Boraston, A. B., Healey, M., Klassen, J., Ficko-Blean, E., Lammerts Van Bueren, A., and Law, V. (2006). A structural and functional analysis of alpha-glucan recognition by family 25 and 26 carbohydrate-binding modules reveals a conserved mode of starch recognition. J. Biol. Chem. 281, 587-598. doi: 10.1074/ jbc.M509958200

Bradford, M. M. (1976). A rapid and sensitive method for the quantitation of microgram quantities of protein utilizing the principle of proteindye binding. Anal. Biochem. 72, 248-254. doi: 10.1016/0003-2697(76)90 527-3

Busi, M. V., Palopoli, N., Valdez, H. A., Fornasari, M. S., Wayllace, N. Z., GomezCasati, D. F., et al. (2008). Functional and structural characterization of the catalytic domain of the starch synthase III from Arabidopsis thaliana. Proteins 70, 31-40. doi: 10.1002/prot.21469
Finally, we evaluated the contribution of the two binding sites in the D2 domain, and observed that the W279A mutation moderately decreased the apparent affinity for glycogen, similarly to the W366 mutation in the D2 domain of $A$. thaliana SSIII. However, the W304A mutation (comparable to Y394A in A. thaliana SSIII D2) caused a 10 -fold decrease in $S_{0.5}$ for glycogen, indicating a greater contribution of binding site 1 in the apparent affinity for polysaccharide substrates.

\section{AUTHOR CONTRIBUTIONS}

JB planned and performed the experiments, analyzed the data, and wrote the manuscript. MBV performed the experiments and analyzed the data. NP performed the structure modeling and analyzed the data. DFG-C, AAI, MAB, and MVB planned the experiments, analyzed the data, and wrote the manuscript.

\section{FUNDING}

This work was supported by CONICET (PIP 0134) and PIOCONICET-YPF (13320140100061CO) and Agencia Nacional de Promoción Científica y Tecnológica (ANPCyT, PICT RAICES 2011-0982; PICT Joven 2012-0981; PICT 2015-1767).

\section{ACKNOWLEDGMENTS}

We thank Dr. Evelyne Derelle (Université Pierre et Marie Curie, Paris 6, France) for kindly providing O. tauri genomic DNA.

Demir-Hilton, E., Sudek, S., Cuvelier, M. L., Gentemann, C. L., Zehr, J. P., and Worden, A. Z. (2011). Global distribution patterns of distinct clades of the photosynthetic picoeukaryote Ostreococcus. ISME J. 5, 1095-1107. doi: 10.1038/ ismej.2010.209

Deschamps, P., Guillebeault, D., Devassine, J., Dauvillee, D., Haebel, S., Steup, M., et al. (2008a). The heterotrophic dinoflagellate Crypthecodinium cohnii defines a model genetic system to investigate cytoplasmic starch synthesis. Eukaryot. Cell 7, 872-880. doi: 10.1128/EC.00461-07

Deschamps, P., Haferkamp, I., D’hulst, C., Neuhaus, H. E., and Ball, S. G. (2008b). The relocation of starch metabolism to chloroplasts: when, why and how. Trends Plant Sci. 13, 574-582. doi: 10.1016/j.tplants.2008.08.009

Deschamps, P., Moreau, H., Worden, A. Z., Dauvillee, D., and Ball, S. G. (2008c). Early gene duplication within chloroplastida and its correspondence with relocation of starch metabolism to chloroplasts. Genetics 178, 2373-2387. doi: 10.1534/genetics.108.087205

Fusari, C., Demonte, A. M., Figueroa, C. M., Aleanzi, M., and Iglesias, A. A. (2006). A colorimetric method for the assay of ADP-glucose pyrophosphorylase. Anal. Biochem. 352, 145-147. doi: 10.1016/j.ab.2006.01.024

Giuliani, S. E., Frank, A. M., and Collart, F. R. (2008). Functional assignment of solute-binding proteins of $\mathrm{ABC}$ transporters using a fluorescence-based thermal shift assay. Biochemistry 47, 13974-13984. doi: 10.1021/bi801648r

Guillén, D., Sánchez, S., and Rodríguez-Sanoja, R. (2010). Carbohydrate-binding domains: multiplicity of biological roles. Appl. Microbiol. Biotechnol. 85, 1241-1249. doi: 10.1007/s00253-009-2331-y

Kanehisa, M., Furumichi, M., Tanabe, M., Sato, Y., and Morishima, K. (2017). KEGG: new perspectives on genomes, pathways, diseases and drugs. Nucleic Acids Res. 45, D353-D361. doi: 10.1093/nar/gkw1092

Kawazu, T., Nakanishi, Y., Uozumi, N., Sasaki, T., Yamagata, H., Tsukagoshi, N., et al. (1987). Cloning and nucleotide sequence of the gene coding for 
enzymatically active fragments of the Bacillus polymyxa beta-amylase. J. Bacteriol. 169, 1564-1570. doi: 10.1128/jb.169.4.1564-1570.1987

Nakamura, Y. (2002). Towards a better understanding of the metabolic system for amylopectin biosynthesis in plants: rice endosperm as a model tissue. Plant Cell Physiol. 43, 718-725. doi: 10.1093/pcp/pcf091

Okonechnikov, K., Golosova, O., and Fursov, M. (2012). Unipro UGENE: a unified bioinformatics toolkit. Bioinformatics 28, 1166-1167. doi: 10.1093/ bioinformatics/bts091

Palopoli, N., Busi, M. V., Fornasari, M. S., Gomez-Casati, D., Ugalde, R., and Parisi, G. (2006). Starch-synthase III family encodes a tandem of three starchbinding domains. Proteins 65, 27-31. doi: 10.1002/prot.21007

Patron, N., and Keeling, P. J. (2005). Common evolutionary origin of starch biosynthetic enzymes in green and red algae1. J. Phycol. 41, 1131-1141. doi: 10.1111/j.1529-8817.2005.00135.x

Penninga, D., Van Der Veen, B. A., Knegtel, R. M., Van Hijum, S. A., Rozeboom, H. J., Kalk, K. H., et al. (1996). The raw starch binding domain of cyclodextrin glycosyltransferase from Bacillus circulans strain 251. J. Biol. Chem. 271, 32777-32784. doi: 10.1074/jbc.271.51.32777

Pfister, B., and Zeeman, S. C. (2016). Formation of starch in plant cells. Cell. Mol. Life Sci. 73, 2781-2807. doi: 10.1007/s00018-016-2250-x

Polekhina, G., Feil, S. C., Gupta, A., O'donnell, P., Stapleton, D., and Parker, M. W. (2005). Crystallization of the glycogen-binding domain of the AMP-activated protein kinase beta subunit and preliminary X-ray analysis. Acta Crystallogr. Sect. F Struct. Biol. Cryst. Commun. 61, 39-42. doi: 10.1107/S1744309104025059

Preiss, J., Ball, K., Smith-White, B., Iglesias, A., Kakefuda, G., and Li, L. (1991). Starch biosynthesis and its regulation. Biochem. Soc. Trans. 19, 539-547. doi: 10.1042/bst0190539

Ral, J. P., Derelle, E., Ferraz, C., Wattebled, F., Farinas, B., Corellou, F., et al. (2004). Starch division and partitioning. A mechanism for granule propagation and maintenance in the picophytoplanktonic green alga Ostreococcus tauri. Plant Physiol. 136, 3333-3340. doi: 10.1104/pp.104.044131

Sali, A. (1995). Comparative protein modeling by satisfaction of spatial restraints. Mol. Med. Today 1, 270-277. doi: 10.1016/S1357-4310(95)91170-7

Sato, N. (2001). SSDB: sequence similarity database in KEGG. Genome Inform. 12, $230-231$.

Southall, S. M., Simpson, P. J., Gilbert, H. J., Williamson, G., and Williamson, M. P. (1999). The starch-binding domain from glucoamylase disrupts the structure of starch. FEBS Lett. 447, 58-60. doi: 10.1016/S0014-5793(99)00263-X
Sticklen, M. (2006). Plant genetic engineering to improve biomass characteristics for biofuels. Curr. Opin. Biotechnol. 17, 315-319. doi: 10.1016/j.copbio.2006. 05.003

Tormo, J., Lamed, R., Chirino, A. J., Morag, E., Bayer, E. A., Shoham, Y., et al. (1996). Crystal structure of a bacterial family-III cellulose-binding domain: a general mechanism for attachment to cellulose. EMBO J. 15, 5739-5751. doi: 10.1002/j.1460-2075.1996.tb00960.x

Valdez, H. A., Busi, M. V., Wayllace, N. Z., Parisi, G., Ugalde, R. A., and GomezCasati, D. F. (2008). Role of the N-terminal starch-binding domains in the kinetic properties of starch synthase III from Arabidopsis thaliana. Biochemistry 47, 3026-3032. doi: 10.1021/bi702418h

Valdez, H. A., Peralta, D. A., Wayllace, N. Z., Grisolía, M. J., GomezCasati, D. F., and Busi, M. V. (2011). Preferential binding of SBD from Arabidopsis thaliana SSIII to polysaccharides: study of amino acid residues involved. Starch Stärke 63, 451-460. doi: 10.1002/star.20100 0111

Wayllace, N. Z., Valdez, H. A., Ugalde, R. A., Busi, M. V., and GomezCasati, D. F. (2010). The starch-binding capacity of the noncatalytic SBD2 region and the interaction between the $\mathrm{N}$ - and C-terminal domains are involved in the modulation of the activity of starch synthase III from Arabidopsis thaliana. FEBS J. 277, 428-440. doi: 10.1111/j.1742-4658.2009.07 495.x

Zeeman, S. C., Kossmann, J., and Smith, A. M. (2010). Starch: its metabolism, evolution, and biotechnological modification in plants. Annu. Rev. Plant Biol. 61, 209-234. doi: 10.1146/annurev-arplant-042809-11 2301

Conflict of Interest Statement: The authors declare that the research was conducted in the absence of any commercial or financial relationships that could be construed as a potential conflict of interest.

Copyright (C) 2018 Barchiesi, Velazquez, Palopoli, Iglesias, Gomez-Casati, Ballicora and Busi. This is an open-access article distributed under the terms of the Creative Commons Attribution License (CC BY). The use, distribution or reproduction in other forums is permitted, provided the original author(s) and the copyright owner(s) are credited and that the original publication in this journal is cited, in accordance with accepted academic practice. No use, distribution or reproduction is permitted which does not comply with these terms. 